\title{
Papillary thyroid carcinoma in a 5-year old child, mimicking lymphoma in presentation
}

\author{
Tariq N. Aladily, Majd Khader, Nadwa Bustami, Osama A. Samara ${ }^{1}$ \\ Department of Pathology, ${ }^{1}$ Department of Radiology and Nuclear Medicine, The University of Jordan, Amman, Jordan
}

\begin{tabular}{|c|}
\hline Access this article online \\
\hline Website: www.avicennajmed.com \\
\hline DOI: 10.4103/ajm.ajm_288_20 \\
\hline Quick Response Code: \\
\hline
\end{tabular}

\begin{abstract}
Thyroid cancer is very rare in children and papillary thyroid carcinoma (PTC) represents the most common type. Patients are frequently in the second decade of life and complain of painless enlargement of the gland. Pediatric PTC has unique clinicopathologic characteristics that make it different from the adult counterpart. The biologic behavior tends to be aggressive and patients frequently present with advanced disease. Herein, we report a case with an unusual presentation. A 5-year-old child manifested with fever, night sweats, cervical lymphadenopathy, and weight loss for 2 months. He also complained of mild cough and shortness of breath. Clinical suspicion of tuberculosis or lymphoma was raised, but laboratory workup was unremarkable. Cervical lymph node excision was done, and the histopathologic examination showed metastatic PTC. The patient underwent surgical and radioactive therapy and remained in complete remission for 5 years. Unfortunately, the disease ultimately relapsed with disseminated metastasis and the patient passed away.
\end{abstract}

Key words: Lymphoma, papillary thyroid carcinoma, pediatric cancer, thyroid carcinoma, tuberculosis

\section{INTRODUCTION}

Cancer in children is rare, and thyroid carcinoma represents less than $3 \%$ of all pediatric malignancies. ${ }^{[1]}$ Papillary thyroid carcinoma is the most common type and its incidence appears to rise in the last four decades, which is related to better detection and accurate diagnosis. Most cases arise during teenage years. Possible risk factors include radiation exposure, iodine deficiency, previous history of Hashimoto's thyroiditis, and certain inherited syndromes. ${ }^{[2]}$ The modality of therapy is extrapolated from adult diseases, as patients undergo surgical excision with further adjuvant radioactive iodine for non-resectable lesions. Targeted kinase inhibitors provide a promising option in refractory disease. ${ }^{[3]}$

Children with PTC typically manifest with an asymptomatic neck mass with or without lymphadenopathy. Associated dyspnea is common, and some patients have an associated hyperthyroidism. Fine needle aspiration of the neck mass is very helpful in establishing the diagnosis, as cytologic

Address for correspondence: Dr Tariq N. Aladily, Department of Pathology, The University of Jordan, Amman, Jordan.

E-mail: tnaladily@ju.edu.jo features of PTC are easy and can be achieved by simple sedation in children. The presence of B-symptoms in pediatric PTC is unusual and would provisionally warrant an alternative diagnosis such as infections or lymphoma.

\section{CASE REPORT}

In June 2014, a 5-year-old boy presented with a 2-month history of fever, night sweats, weight loss, and cough. Physical examination revealed bilateral anterior cervical lymph nodes enlargement which were fixed and painless. The patient also had peripheral cyanosis in digits. Complete blood count test showed hemoglobin concentration: $128 \mathrm{~g} / \mathrm{L}$, white blood cell count: $9.12 \times 10^{9} / \mathrm{L}$ (30.1\% neutrophils, $57.3 \%$ lymphocytes,

This is an open access journal, and articles are distributed under the terms of the Creative Commons Attribution-NonCommercial-ShareAlike 4.0 License, which allows others to remix, tweak, and build upon the work non-commercially, as long as appropriate credit is given and the new creations are licensed under the identical terms.

For reprints contact: reprints@medknow.com

Cite this article as: Aladily TN, Khader M, Bustami N, Samara OA. Papillary thyroid carcinoma in a 5-year old child, mimicking lymphoma in presentation. Avicenna J Med 2021;11:103-6. 
6.61\% eosinophils, $4.54 \%$ monocytes, $1.45 \%$ basophils) and platelet count: $329 \times 10^{9} / \mathrm{L}$. Liver enzymes, serum bilirubin, creatinine, and blood urea nitrogen were all normal. Blood culture showed no bacterial growth.

Chest X-ray revealed bilateral airway narrowing. Neck computed tomography (CT) scan showed multiple bilateral anterior cervical as well as supraclavicular and infraclavicular lymphadenopathy causing mass effect. The largest lymph node measured was $20 \times 15 \mathrm{~mm}$. Some lymph nodes showed cystic degeneration suggestive of central necrosis. The thyroid gland exhibited heterogenous density. CT scan of the chest demonstrated bilateral nodular and micronodular lung densities and hilar lymphadenopathy [Figures 1-3]. Abdominal CT scan showed mild hepatomegaly and paraaortic lymph node enlargement. The overall clinical impression was favoring either tuberculosis or Hodgkin's lymphoma (HL).

Based on the clinical suspicion of lymphoma, an excisional biopsy of a single cervical lymph node was performed. A bone marrow trephine biopsy was also obtained for staging. Histopathological examination of the lymph node showed proliferation of malignant thyroid glands, demonstrating papillary growth and characteristic nuclear clearing and grooves of follicular epithelial cells. Only a residual rim of lymph node tissue was present [Figures 4-6]. The diagnosis of metastatic papillary thyroid carcinoma was established. The bone marrow biopsy was negative for metastasis. The patient was in clinical stage-I disease.

The patient was transferred to another facility and had total thyroidectomy and cervical lymph nodes excision. Then he received radioactive iodine therapy. The patient remained in complete remission until December 2019 when he developed respiratory symptoms. CT scan revealed multiple bilateral pulmonary nodules consistent with metastasis. The patient had many admissions to the intensive care unit for

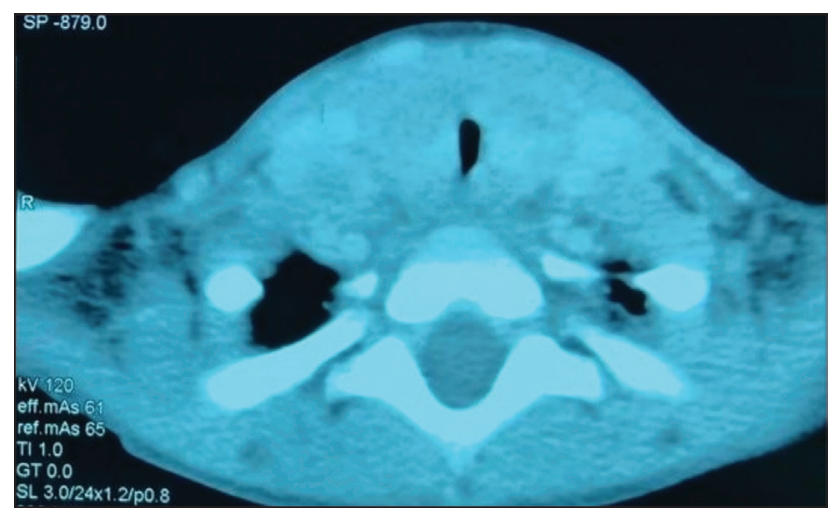

Figure 1: Neck CT without contrast showing diffuse thyroid enlargement, bilateral lymphadenopathy, and compression to trachea unstable vital signs. He died in March 2020 of septicemia by methicillin-resistant Staphylococcus aureus.

\section{DISCUSSION}

Pediatric PTC remains an uncommon disease despite recent annual increase in incidence. The disease is more common in females and only one-third of the patients are younger than 12 years. PTC in children and adolescents represents a biologically and molecularly distinct disease that is different from adults counterpart. For example, BRAF genetic mutation is very rare in pediatric PTC, while expression of sodium-iodide transporter in metastatic foci is common compared with adults. ${ }^{[2,4]}$ The traditional prognostic variables of sex, disease stage, relapse, and histologic subtype do not have a similar impact on outcome as in adult disease. When the disease arises in adolescents, the outcome is comparable to adults. However, PTC in prepubertal age shows inferior prognosis. ${ }^{[5]}$

The identification of a thyroid nodule in adults is common and harbors a low incidence of malignancy. In contrast, children rarely develop thyroid nodules, and the chance

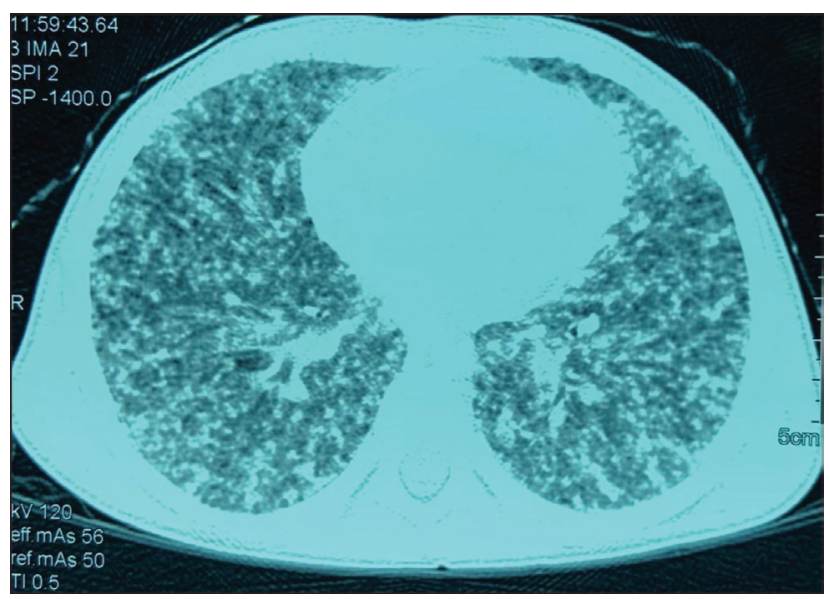

Figure 2: Chest HRCT scan showing innumerable micronodules in both lungs of miliary pattern of dissemination

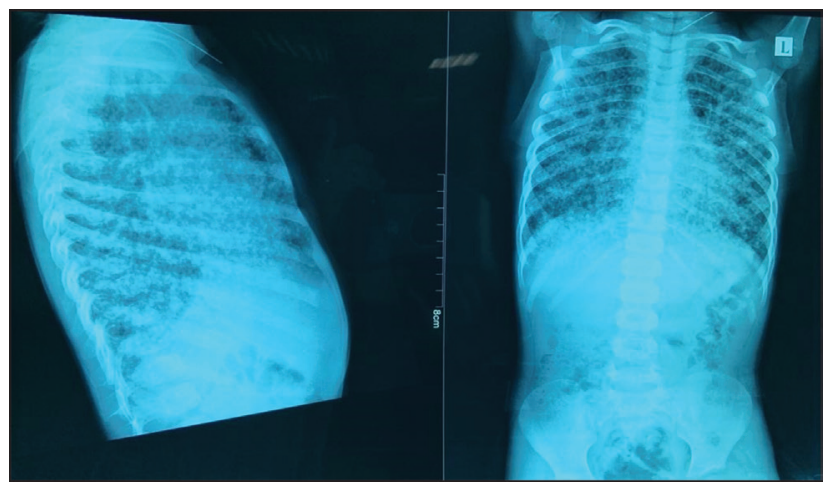

Figure 3: Chest-X-ray (posterioanterior and lateral views) showing diffuse bilatera miliary nodular opacities 


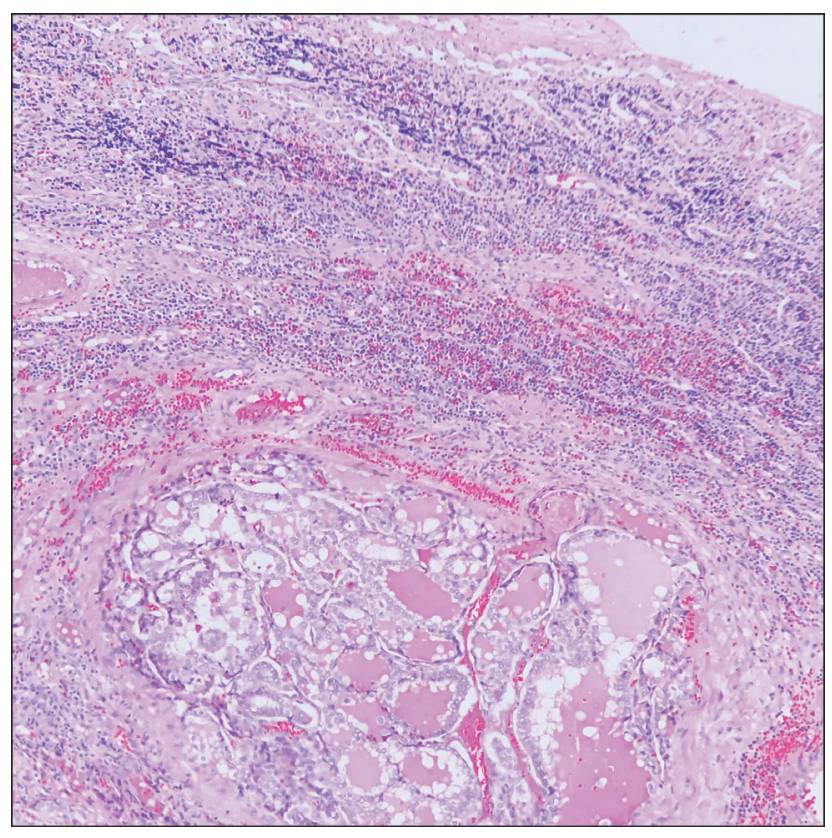

Figure 4: Cervical lymph node excision: low-power view showing a residual rim of lymphoid tissue (top) and presence of thyroid glands (below)

of malignancy is as high as a quarter of cases. ${ }^{[2]}$ Clinical features suggestive of malignancy are, if the nodule is large and hard, adherent to neck structures or associated with lymphadenopathy. Given the small size of thyroid gland in children, pediatric PTC tends to have larger size, greater chance of invasion to adjacent structures, and distant metastasis compared with adults. The rate of recurrence is also high. ${ }^{[1]}$

The constellation of fever, night sweats, and unintentional weight loss, which are referred to as B-symptoms, are characteristics of certain diseases that are primarily chronic and have an inflammatory nature. Prolonged fever is alarming for an infectious or neoplastic disease. Neoplastic fever is commonly non-infectious and occurs secondary to elevated cytokines levels. Mutant genes in neoplastic cells are responsible for aberrant release of cytokines. In addition, tumor-induced inflammation, tissue damage, and deep vein thrombosis would promote this condition. High levels of cytokines induce prostacyclin release which directly affects hypothalamus to change its thermostatic function. ${ }^{[6]}$

$\mathrm{HL}$ is the most common type of lymphoma in children. ${ }^{[7]}$ It primarily affects cervical lymph nodes and spreads contiguously to mediastinal lymph nodes, causing dyspnea and cough ${ }^{[8]}$ Approximately 25\% of the HL patients suffer from B-symptoms secondary to high level of interleukin-6. ${ }^{[6]}$ In addition, infection with tuberculosis would cause respiratory symptoms, fever, and commonly cervical lymphadenopathy. Thus, the symptoms of the patient we present would strongly match with both diseases.

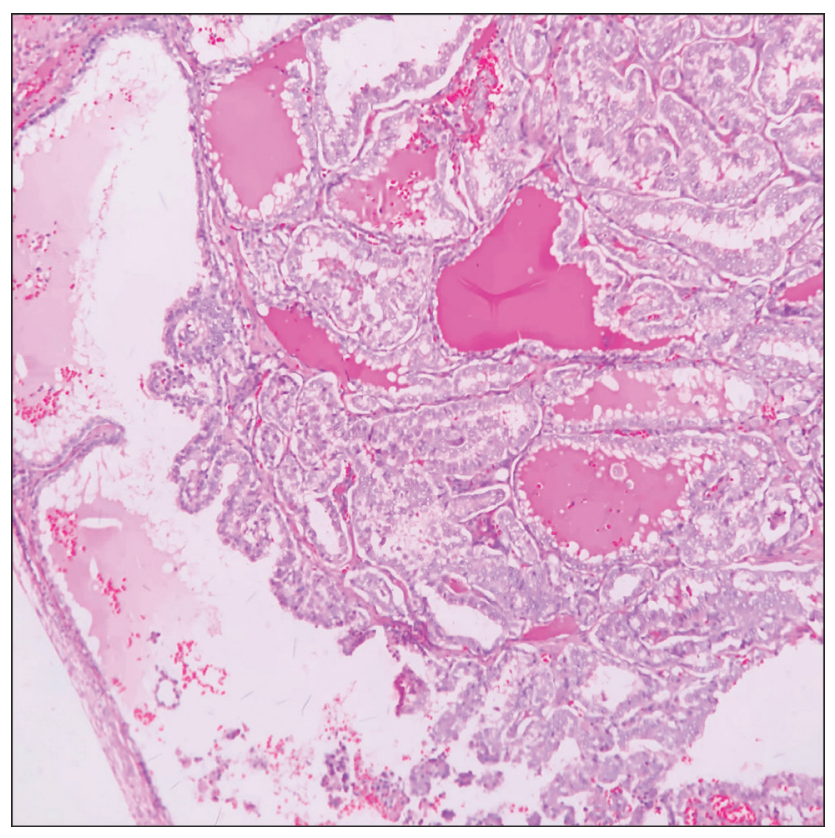

Figure 5: Medium power view: follicular epithelial cells are arranged in papillae harboring fibrovascular cores, most notably on the left side of this view

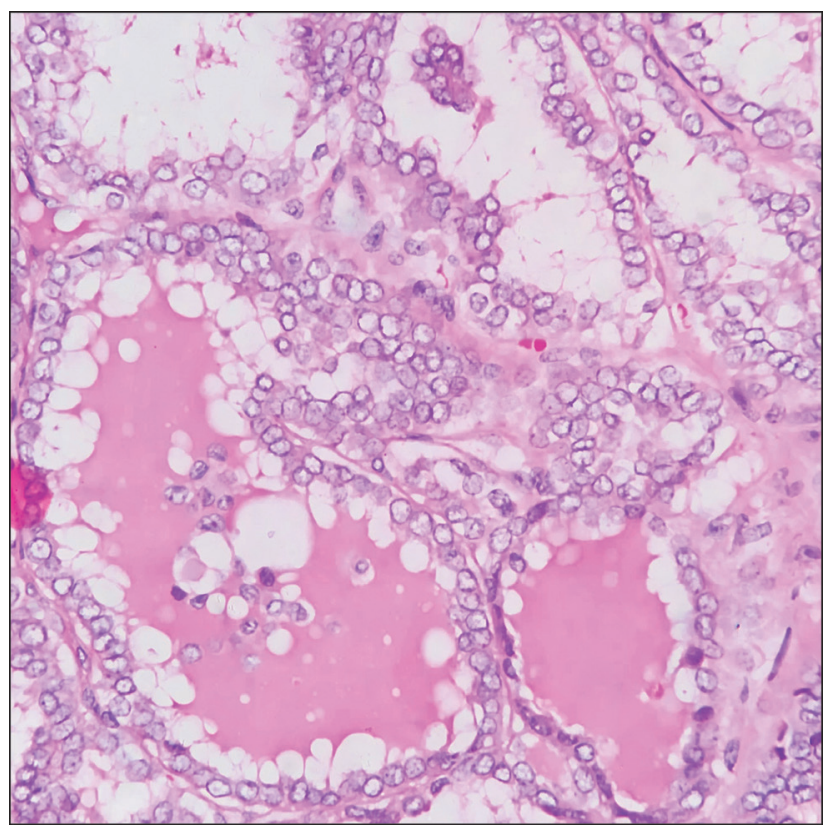

Figure 6: High power view: follicular epithelial cells show prominent nuclear clearing "Orphan Anne" and occasional nuclear grooves, characteristic of papillary thyroid carcinoma

In addition to lymphoma, B-symptoms were described in several hematologic and solid tumors such as acute leukemia, renal cell carcinoma, hepatocellular carcinoma, ovarian cancers, and glioblastoma multiforme. ${ }^{[6]}$ The emergence of B-symptoms in pediatric PTC is exceptionally rare and, to the best of our knowledge, was not described previously. Despite the presence of respiratory symptoms, no definite infectious cause was identified and B-symptoms disappeared after surgical intervention. 


\section{CONCLUSION}

This case demonstrates the need to consider PTC in the differential diagnosis of children presenting with neck mass and fever, despite its rare incidence. It also highlights the important role of histopathologic evaluation to reach the correct diagnosis.

\section{Financial support and sponsorship}

Nil.

\section{Conflicts of interest}

There are no conflicts of interest.

\section{RefEREnces}

1. Vaisman F, Corbo R, Vaisman M. Thyroid carcinoma in children and adolescents-Systematic review of the literature. J Thyroid Res 2011;2011:845362.
2. Paulson VA, Rudzinski ER, Hawkins DS. Thyroid cancer in the pediatric population. Genes (Basel) 2019;10:723. doi:10.3390/genes10090723

3. Verburg FA, Van Santen HM, Luster M. Pediatric papillary thyroid cancer: Current management challenges. Onco Targets Ther 2017;10:165-75

4. Patel A, Jhiang S, Dogra S, Terrell R, Powers PA, Fenton C, et al. Differentiated thyroid carcinoma that express sodium-iodide symporter have a lower risk of recurrence for children and adolescents. Pediatr Res 2002;52:737-44.

5. Lazar L, Lebenthal Y, Steinmetz A, Yackobovitch-Gavan M, Phillip M. Differentiated thyroid carcinoma in pediatric patients: Comparison of presentation and course between pre-pubertal children and adolescents. J Pediatr 2009;154:708-14.

6. Foggo V, Cavenagh J. Malignant causes of fever of unknown origin. Clin Med (Lond) 2015;15:292-4.

7. Aladily TN, Khreisat W, Ashukhaibi O, Alkhatib SM, Annab H, Tarawneh MS, et al. The epidemiology of lymphoma in Jordan: A nationwide population study of 4189 cases according to World Health Organization classification system [published online ahead of print, 2020 Nov 11]. Hematol Oncol Stem Cell Ther 2020;S16583876(20)30171-0. doi:10.1016/j.hemonc.2020.10.002

8. Aladily TN. Circulating Hodgkin cells: A clinicopathologic analysis of seven patients and a review of the literature [published online ahead of print, 2020 Sep 23]. Int J Lab Hematol2020. doi:10.1111/ijlh.13344 